\title{
Variations of the Superficial Palmar Arch in a Sri Lankan Perspective: A Cadaveric Study
}

\author{
Salgado L S S, Hasan R, Perera A A M M S L, Wijesundara W M R D, Anuradha W K
}

Abstract-

Introduction

The blood supply of the hand is a complex and challenging area of study. The clinical importance of this area lies in the significant number of surgical procedures such as radial artery conduits in coronary artery bypass grafting (CABG), radial arterial cannulation and reconstructive surgery of the hand. Anatomical variations in the typical blood supply of the hand are common and are an area of extensive research.

\section{Methodology-}

This descriptive study was carried out in the dissecting theatres at the Department of Anatomy, Faculty of Medicine, University of Kelaniya on dissections carried out on 25 cadaveric hands.

Results-

From the superficial palmar arches studied the following results were obtained. $88 \%$ were complete while $22 \%$ were incomplete. Specimens with contribution from both the radial and the ulnar arteries for the superficial palmar arches were $80 \%$, while specimens with the contribution solely from the ulnar artery were $12 \% .8 \%$ hands studied had contributions from the radial, ulnar and the median arteries. Three branches of the radial artery contribute to complete the superficial palmar arch. Of this $60 \%$ cadavers had the major contribution from the superficial palmar branch of the radial artery, $24 \%$ from the arteria princeps pollicis, and $16 \%$ from the first dorsal metacarpal artery. The contribution to the arterial supply of the radial side of the thumb was from the superficial palmar branch of radial artery in $80 \%$ of the cadavers while in $20 \%$ the contribution was from the arteria princeps pollicis. The contribution to the arterial supply of the ulnar side of the thumb was from the proper palmar digital artery arising from the superficial palmar arch in $44 \%$ hands and the contribution in $32 \%$ hands was from the arteria princeps pollicis while the contribution in $24 \%$ hands was from the first dorsal metacarpal artery.

\section{Conclusion}

It is thus evident that significant anatomical variations of the superficial palmar arch exist in the Sri Lankan population. The arterial supply of the thumb also had significant changes compared to the descriptions given in standard text books.
Keywords- superficial palmar arch; thumb; arterial supply

\section{INTRODUCTION}

The anatomy of the hand is of significant interest to various disciplines of science. The arterial supply of the human hand is of major importance given its clinical relevance. A thorough knowledge of the arterial supply of the hand is imperative when carrying out procedures such as radial artery conduits in coronary artery bypass grafting $(\mathrm{CABG})$, radial arterial cannulation and reconstructive surgery of the hand. The hand is supplied by the terminal branches of the radial \& ulnar arteries. The blood supply of the hand is derived from the superficial and the deep palmar arches (respectively SPA and DPA). These are shown in figure 1 and 2 . There are two types of superficial palmar arches. They are the complete palmar arch and the incomplete palmar arch.

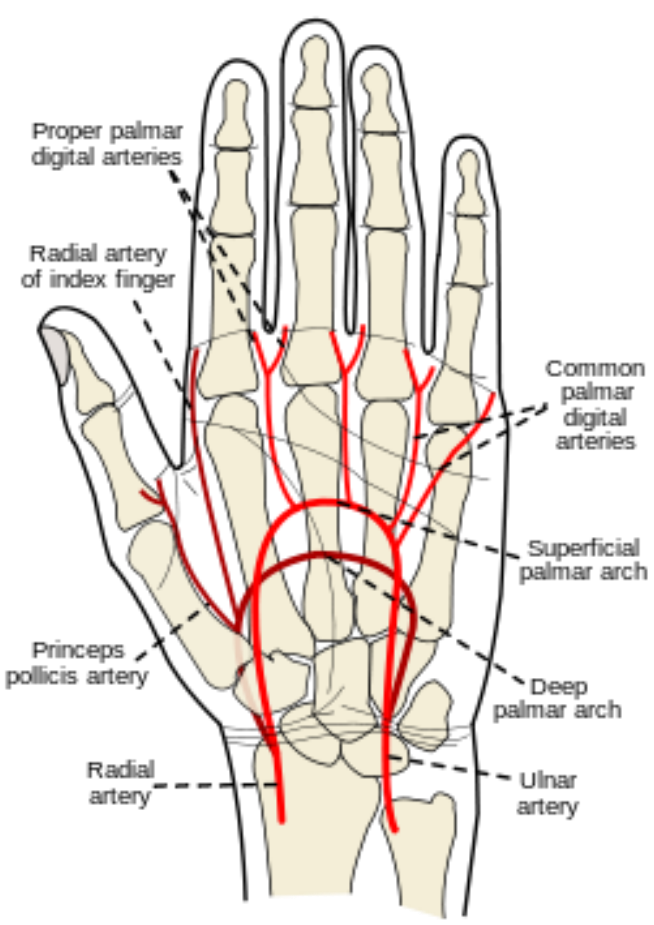

Word count- 311
Figure 1- Deep Palmar Arch ( Picture courtesy ofhttp://en.wikipedia.org/wiki/Deep_palmar_arch ) 


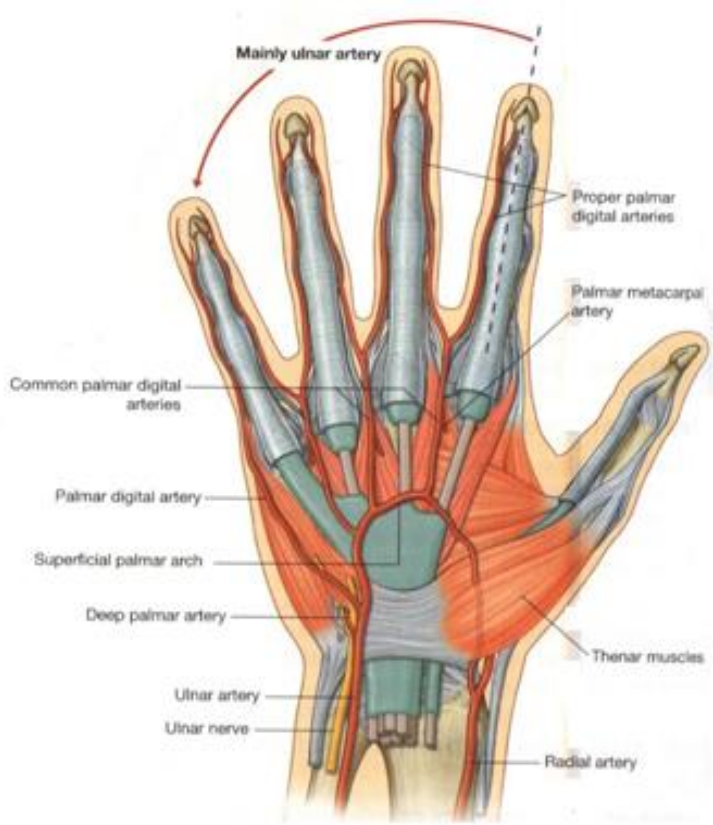

Figure 2- Superficial Palmar Arch (Picture courtesy of Grays Anatomy for Students $2^{\text {nd }}$ ed. https://www.inkling.com/read/gray-anatomy-studentsdrake-vogl-mitchell-2nd/chapter-7/figure-7-106)

\section{A. The course and branches of the radial artery in the hand}

The radial artery enters the palm through the upper end of the first metacarpal space between the heads of the first dorsal interosseus muscle. In the palm it lies between this muscle and the adductor pollicis. It anastomoses with the deep palmar (volar) branch from the ulnar artery to continue as the deep palmar arch. At the wrist the radial artery gives off two branches, namely the dorsal carpal artery and the first dorsal metacarpal artery. The arteria princeps pollicis arises from the radial just as it turns medial ward to the deep part of the hand. It descends along to the ulnar side of the metacarpal bone of the thumb to the base of the first phalanx, where it divides into two branches. These appear between the medial and lateral insertions of the adductor pollicis obliquus and form an arch over the palmar surface of the last phalanx from which arise branches to the integument and subcutaneous tissues of the thumb. The radialis indicis artery is another branch of the radial artery. It anastomoses with the proper digital artery to supply the ulnar side of the index finger. At the lower border of the adductor pollicis transversus this vessel anastomoses with the princeps pollicis and gives a communicating branch to the superficial palmar arch. The deep palmar arch is formed by the anastomosis of the terminal part of the radial artery and the deep palmar branch of the ulnar artery. It gives off the palmar metacarpal arteries, the perforating branches and the recurrent branches.

\section{B. The course and branches of the ulnar artery in the hand}

The ulnar artery begins at the neck of the radius and descends to the wrist. Just above the wrist it becomes superficial between the tendons of the flexor carpi ulnaris and the flexor digitorum superficialis. It pierces the deep fascia proximal to the flexor retinaculum and gives off its deep palmar branch. (This anastomoses with the radial artery) The ulnar artery continues as the superficial palmar arch. In the wrist the ulnar artery also gives off the volar carpal branch, also known as the palmar carpal branch or the anterior ulnar carpal artery and the dorsal carpal branch also known as the posterior ulnar carpal artery. The superficial plantar arch is thus formed by the ulnar artery and usually completed by a branch from the arteria volaris indicis radialis more commonly known as the radialis indicis artery of the radial artery. It is however sometimes completed by the superficial volar branch also known as the superficial palmar artery which arises from the radial artery in the forearm. In some instances the superficial palmar arch has been found to be completed by the arteria princeps pollicis of the radial artery [1].

\section{The superficial palmar arch}

The superficial palmar arch is an arterial arcade which lies adjacent to the palmar aponeurosis. The arch begins on the flexor retinaculum immediately distal to the pisiform bone; it crosses the hook of the hamate deep to the Palmaris brevis. It turns laterally and pierces the medial septum of the palm. It continues deep to the palmar aponeurosis to anastomose with branches of the radial artery as mentioned above. The superficial palmar arch has many branches the main ones being the four digital arteries [2]. The superficial palmar arch can be complete or incomplete. Incomplete superficial palmar arches are clinically important because individuals who have them are at increased risk of digital ischemia. In a study by Coleman et al., the complete arch was found in $78.5 \%$ of the cases and incomplete arch in the remaining $21.5 \%$ [3]. A study conducted by Ikeda et al. using 220 cadaveric specimens identified the complete superficial palmar arch in $96.4 \%$ of the cases and an incomplete palmar arch in 3.6\% [4]. Gellman et al. showed a complete superficial palmar arch in $84.4 \%$ of cases [5], while $\mathrm{Al}$ Turk and Metcalf also reported complete superficial palmar arch in $84 \%$ of the cases [6]. According to Williams P et al, about one - third of the SPA is formed by the ulnar artery alone; a further third is completed by the superficial palmar branch of the radial artery and a third either by the arteria radialis indicis or by the princeps pollicis or by the median artery (The median artery is a branch of the anterior interosseous artery which is a branch from the ulnar artery. The median artery arises in the forearm and accompanies the median nerve) [1]. Furthermore this study states that the classical type of the superficial palmar arch in which the superficial branch of the ulnar artery anastomoses with the superficial branch of the ulnar artery is found in only $34.5 \%$ of cases. In 2009 a study was done by Bataineh et al using 30 formalin fixed cadaveric hands. A rare case was reported in this study in which an incomplete superficial palmar arch was formed by the median artery which gave rise to the princeps pollicis and radialis indicis arteries [7]. 
In 2005 Loukas et al mentioned that in $40 \%$ the superficial palmar arch anastomosis of the superficial volar branch of the radial artery to the ulnar artery. In $35 \%$ the superficial palmar arch is formed entirely of the ulnar artery. In $15 \%$ it is formed by anastomosis of the ulnar and median arteries. In $6 \%$ the arch is formed by anastomosis of the ulnar, radial, and median arteries and in $4 \%$ it is formed by a branch of the deep palmar arch [8]. Ozkus $\mathrm{K}$ et al performed a similar study using 80 cadaveric hands. According to them $97.5 \%$ had a superficial palmar arch, $80 \%$ of which were formed by anastomoses between ulnar and radial arteries. In $17.5 \%$ the arch was formed by the ulnar artery alone. In $2.5 \%$ there were no superficial palmar arch and these specimens were supplied by the anterior median artery [9].

\section{The arterial supply of the thumb}

Textbooks in anatomy often mention the fact that the arterial supply of the thumb is derived from the princeps pollicis artery of the radial artery. However intraoperative findings often present with conflicting variations. Ames E L et al carried out a study using 40 cadavers. According to them the most common pattern was the superficial and deep vessels to the first web space in $54 \%$ of specimens. Dominant vessels include the superficial palmar branch of the radial artery in $8 \%$, first palmar metacarpal artery in $18 \%$ and dorsal metacarpal artery in $8 \%$. Only three specimens in their study correlated with the textbook description [10].

\section{E. The importance of knowledge on palmar vascular patterns in the local population}

From these numerous studies it is clear that anatomical variations exist in the vasculature of the hand. From clinical experience it is known that these variations are of significant importance, not only during complex surgeries involving the hand but also in microsurgical techniques of the hand. But it is yet not known whether these changes are associated with race although it has been suggested that variations of the palmar arches are associated with handedness [11]. Vascular studies of the thumb similarly show results which conflict with the textbook description. Whether these results hold true for the Sri Lankan population is still unknown. Given the large number of traumatic injuries of the hand due to occupational injuries occurring in the working class population of Sri Lanka a good knowledge on local patterns of hand vasculature is of paramount importance.

\section{OBJECTIVES}

The objectives of this study are to describe the variations of the superficial palmar arch in a Sri Lankan population and to study the variations in the arterial supply of the thumb in a Sri Lankan population.

\section{METHODOLOGY}

\section{A. Authors and Affiliations}

This descriptive cross sectional study was carried out at the Department of Anatomy, Faculty of Medicine, University of Kelaniya, utilizing dissections of cadavers in the department. The study incorporated only cadavers of adult Sri Lankans which were preserved in $8 \%$ formalin. Only those cadavers with no abnormalities of the hand were included in this study. 25 hands were used for this study including 12 right hands and 13 left hands. While bilateral hands from 2 cadavers were studied the rest were unilateral hands. It is expected that this sample of specimens obtained for this study will represent the vasculature patterns of the Sri Lankan population. The gross dissections were carried out using standard dissection procedures. During the dissections the presence or the absence of the superficial palmar arch was noted. An arch was considered to be complete if an anastomosis was found between the vessels constituting it. An incomplete arch had an absence of a communication or anastomosis between the vessels constituting the arch. The vessels contributing to the formation of the arch were also identified. The thumb muscles were identified and the blood vessels which supplied the muscles of the thumb were also identified. The data was analyzed using SPSS.

\section{RESULTS}

\section{a) Percentages of complete and incomplete superficial palmar arches}

All the specimens had a superficial palmar arch. From all the superficial palmar arches which were dissected $88 \%$ (22) were complete superficial palmar arches (complete SPA) while 12\% (3) were incomplete palmar arches (incomplete SPA). This is shown in Figure 3.

Percentages of the prevalence of complete and incomplete superficial palmar arches as obtained in other studies are variable. These are compared in Table 1.

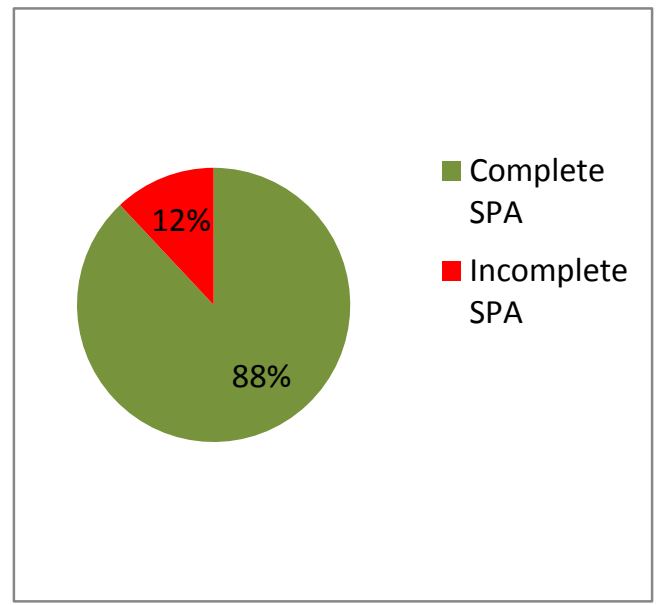

Figure 3- Percentages of types of SPA 
Table 1- Percentages of types of SPA

\begin{tabular}{|l|l|l|}
\hline Name of study & $\begin{array}{l}\text { Complete } \\
\text { superficial } \\
\text { palmar arch }\end{array}$ & $\begin{array}{l}\text { Incomplete } \\
\text { superficial } \\
\text { palmar arch }\end{array}$ \\
\hline Gray's Anatomy[1] & 66.7 & 33.3 \\
\hline Bilge O et al.[12] & 86 & 14 \\
\hline $\begin{array}{l}\text { Ruengsakuirach et } \\
\text { al.[13] }\end{array}$ & 66 & 34 \\
\hline Coleman et al.[3] & 78.5 & 21.5 \\
\hline Ikeda et al.[4] & 96.4 & 3.6 \\
\hline
\end{tabular}

b) The major arteries that contribute in forming the superficial palmar arch

Contribution to the superficial palmar arch from the superficial palmar branches from both the radial (RA) and ulnar arteries (UA) was $80 \%$ (20), while contribution from ulnar artery alone was $12 \%$ (3). Contribution from all three arteries including branches from the ulnar, radial and median artery (MA) which was a branch of the anterior interosseus artery from the ulnar artery during its course in the forearm was only $8 \%$ (2). These are shown in Figure 4.

The percentage of radio- median- ulnar type of superficial palmar arches shows great variation as shown in Table 2. The major arteries which contribute in forming the superficial palmar arch are described differently in various researches. These are compared below in Table 3 .

One of the radio - median - ulnar types of superficial palmar arches that we found during dissections is shown below in Figure 5. The superficial palmar branch of the radial artery (SPBRA) is also shown here.

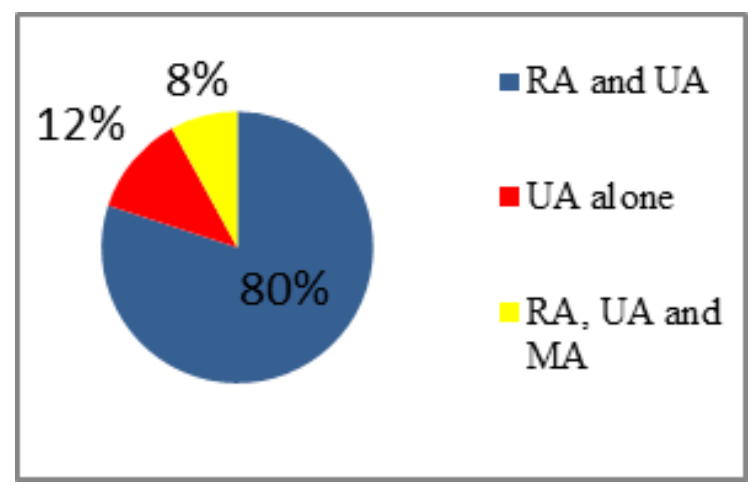

Figure 4- Percentages of the contribution from the major arteries which form the SPA
Table 2- Comparison of percentages of radio- medianulnar type of SPA in researches

\begin{tabular}{|l|l|}
\hline Name of study & $\begin{array}{l}\text { Presence of radio-median- } \\
\text { ulnar type superficial } \\
\text { palmar arch }\end{array}$ \\
\hline D'Costa et al. & $15.8 \%$ \\
\hline Ruengsakuirach et.al[13] & $2 \%$ \\
\hline
\end{tabular}

Table 3- Comparison of percentages of major arteries which form the SPA in researches

\begin{tabular}{|l|l|l|l|}
\hline $\begin{array}{l}\text { Name of } \\
\text { study }\end{array}$ & $\begin{array}{l}\text { Radial and } \\
\text { ulnar }\end{array}$ & $\begin{array}{l}\text { Radial, } \\
\text { median and } \\
\text { ulnar }\end{array}$ \\
\hline $\begin{array}{l}\text { Loukas et al } \\
{[8]}\end{array}$ & $40 \%$ & $35 \%$ & $6 \%$ \\
\hline $\begin{array}{l}\text { Ozkus K et al } \\
{[9]}\end{array}$ & $80 \%$ & $17.5 \%$ & - \\
\hline
\end{tabular}

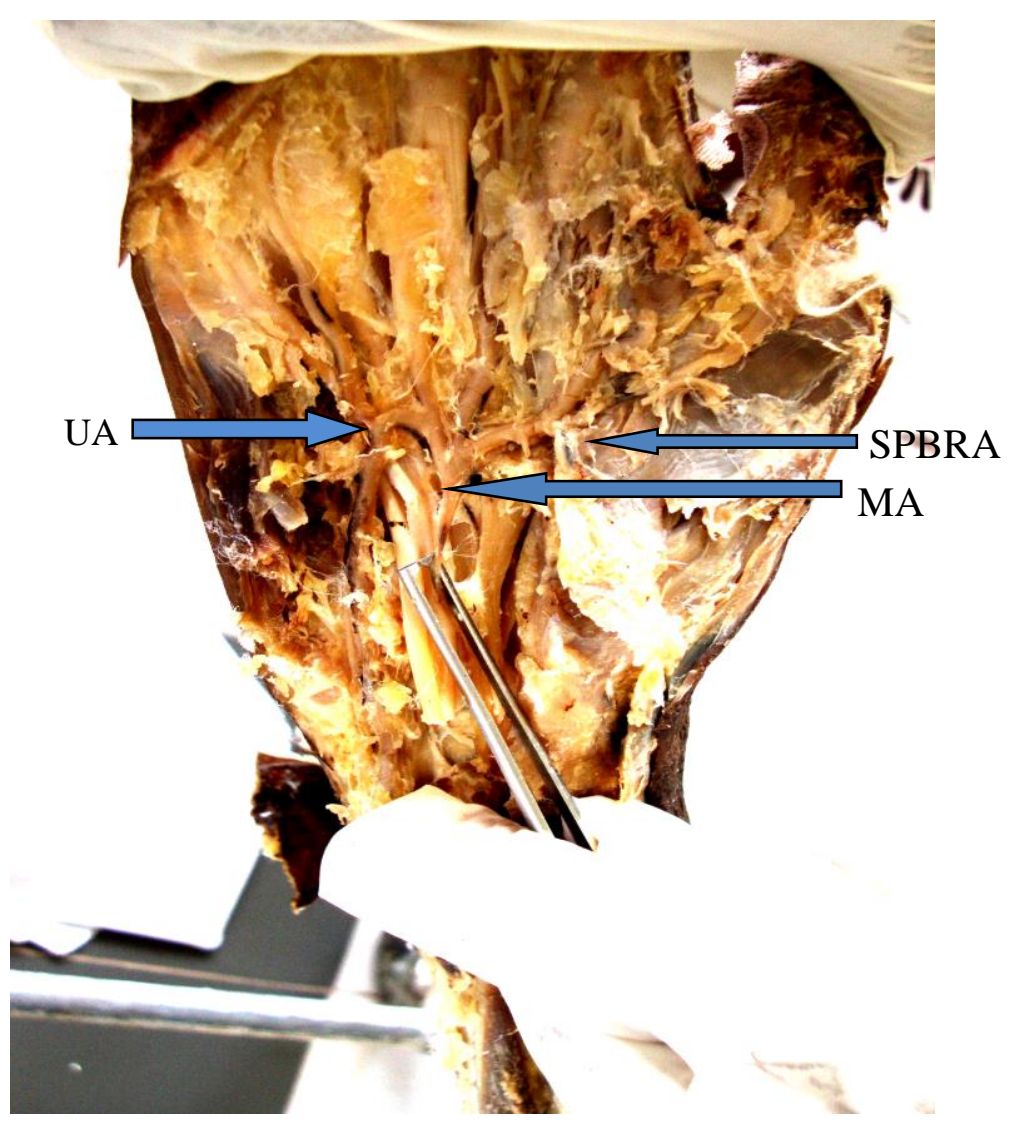

Figure 5- A radio- ulnar- median type of SPA 
c) The branches of the radial artery which form the superficial palmar arch

From the three major branches of the radial artery which can contribute to form the superficial palmar arch, the superficial palmar branch of the radial artery (SPBRA) made the major contribution being present in 60\% (15) of the dissections. Arteria princeps pollicis (APP) and arteria radialis indicis (ARI) completed the superficial palmar arch in $24 \%$ (6) of the dissections while the first dorsal metacarpal artery (FDMA) completed the superficial palmar arch in $16 \%$ (4) of the dissections. These are shown in Figure 6.

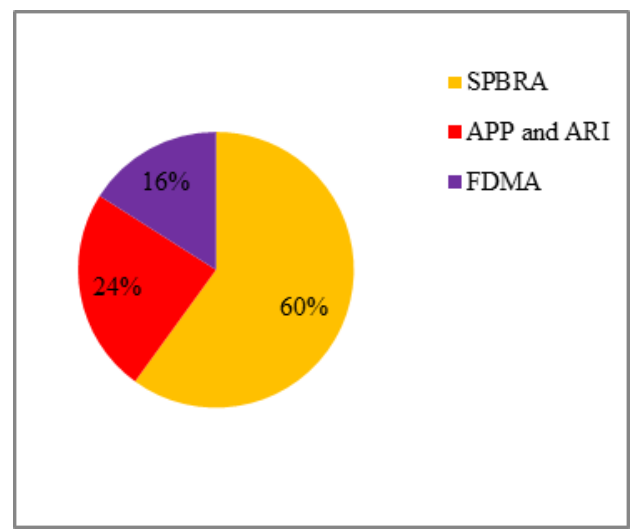

Figure 6- Branches from the radial artery which form the SPA

The first dorsal metacarpal artery as seen during our dissections is shown below in Figure 7. Percentages of completion of the superficial palmar arch by the first dorsal metacarpal artery are compared below in Table 4 .

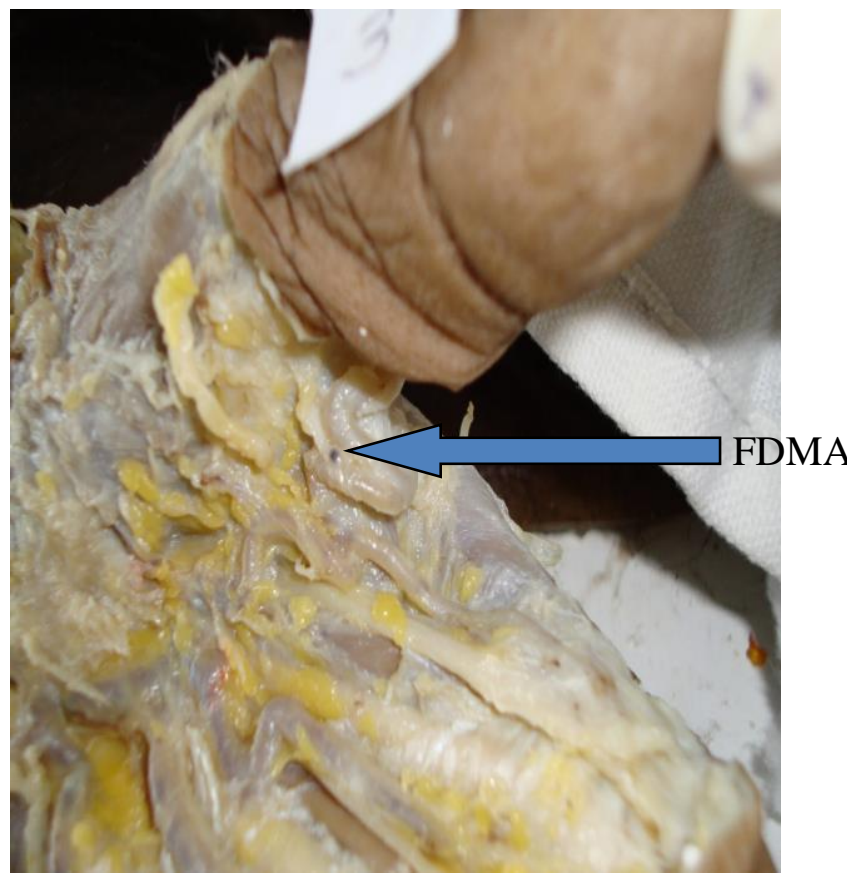

Figure 7- FDMA as seen during our dissections
Table 4- Comparison of percentages of completion of the SPA by the FDMA in various researches

\begin{tabular}{|c|c|}
\hline Name of study & $\begin{array}{r}\text { Percentage of completion of } \\
\text { SPA by FDMA }\end{array}$ \\
\hline Bilge O et al. [12] & $28 \%$ \\
\hline Ruengsakuirach et.al [13] & $18 \%$ \\
\hline
\end{tabular}

d) The arterial supply of the thumb

The arterial supply of the thumb was observed to be different on the radial and the ulnar sides. The radial side was supplied by superficial palmar branch of radial artery (SBPRA) and the arteria princeps pollicis (APP) in respectively $80 \%$ (20) and $20 \%$ (5) of the specimens. The ulnar side was supplied by the proper palmar digital artery from the superficial palmar arch (PPDA from SPA), arteria princeps pollicis, and the first dorsal metacarpal artery (FDMA) on respectively $44 \%$ (11), $32 \%$ (8) and $24 \%$ (6) of the specimens. The arteria princeps pollicis was present in all the specimens. Percentages are given below in Table 5 and Table 6 .

Table 5- Blood supply to the radial side of the thumb

\begin{tabular}{|l|l|l|}
\hline & $\begin{array}{l}\text { Percentage of } \\
\text { supply by SPBRA }\end{array}$ & $\begin{array}{l}\text { Percentage of } \\
\text { supply by APP }\end{array}$ \\
\hline Radial side & $80 \%$ & $22 \%$ \\
\hline
\end{tabular}

Table 6- Blood supply to the ulnar side of thumb

\begin{tabular}{|c|c|c|c|}
\hline & $\begin{array}{c}\text { Percentage of } \\
\text { supply by } \\
\text { PPDA }\end{array}$ & $\begin{array}{c}\text { Percentage of } \\
\text { supply by } \\
\text { APP }\end{array}$ & $\begin{array}{c}\text { Percentage of } \\
\text { supply by } \\
\text { FDMA }\end{array}$ \\
\hline Ulnar side & $44 \%$ & $32 \%$ & $24 \%$ \\
\hline
\end{tabular}

\section{DISCUSSION}

According to our study the majority of the cadavers had a complete superficial palmar arch suggesting that the majority of the Sri Lankan population has a complete superficial palmar arch. In fact the figures obtained in our study indicate that the presence of the complete superficial palmar arch in the Sri Lankan population may be higher than in other races or in other parts of the world. Whether this has an association with the incidence of digital ischemia following hand trauma or surgery is not known. It is also not known whether the incidence of the complete superficial palmar arches correlates with the handedness in the Sri Lankan population. According to our study the majority of the superficial palmar arches were formed by contributions from both the ulnar and radial arteries. $11 \%$ however were supplied by the ulnar artery alone. The superficial palmar arch is an important anastomosis with the major contribution from the ulnar artery. Ulnar arterial 
occlusion either acute or chronic, will seriously threaten the viability of the structures of the palm. A good anastomosis with many feeding vessels will ensure the viability of the tissues of the palm in case of such an event. Our study revealed that $6 \%$ of the cadavers had a superficial palmar arch supplied purely by the ulnar artery. This is a fact worth remembering not only when carrying out the Allen test but also when ligating arteries during palmar surgeries. According to this study a little more than half of the specimens had the superficial palmar arch completed by the superficial palmar branch of the radial artery contrary to the expected finding of the radialis indicis artery of the radial artery as mentioned in textbooks of anatomy. The arterial supply of the thumb is described in most textbooks as derived from the arteria princeps pollicis of the radial artery. Numerous studies have proved that many conflicting variations exist. Our study was able to determine the local patterns of vasculature of the thumb. According to this study the radial side and the ulnar side of the thumb had different blood supply. In the majority of the specimens the radial and ulnar sides of the thumb respectively had their blood supply from the superficial palmar branch of the radial artery and the proper palmar digital artery. This rich and complex anastomosis present in the thumb allows it to survive with minimal damage even in the face of severe arterial insult. Whether this variation is an adaptation to ensure better protection to the most unique finger of the human hand is not known.

\section{CONCLUSION}

From our results it is clear that important variations exist in the macroscopic anatomy of the superficial palmar arch and the blood supply of the thumb. Some of these are compatible with the findings presented in similar studies, but the majority of findings are unique.

\section{ACKNOWLEDGMENT}

This research article is made possible through the help and support of the academic and nonacademic staff of the
Department of Anatomy, Faculty of Medicine, University of Kelaniya. Special thanks also go to the families of the authors who gave immense support and advice.

\section{REFERENCES}

[1] P. L. Willams, L. G. Bannister, M. M. Berry, Gray's Anatomy, 38th ed., New York: Churchill Livingstone; 2000.

[2] G.J. Romanes, Cunningham's manual of practical Anatomy, $13^{\text {th }}$ ed. vol 1. Oxford: Oxford University press, 1986, pp. 95

[3] S. S. Coleman, B. J. Anson, "Arterial patterns in the hand based upon a study of 650 specimens," SurgGynecol Obstet., vol. 113, 1961, pp. 40924. [PubMed]

[4] A. Ikeda, A. Ugawa, Y. Kazihara, N. Hamada, "Arterial patterns in the hand based on a three-dimensional analysis of 220 cadaver hands," J Hand Surg Am., vol. 13, 1988, pp. 501-9. [PubMed])

[5] H. Gellman, M. J. Botte, J. Shankwiler, R. H. Gelberman, “Arterial patterns of the deep and superficial palmar arches," ClinOrthopRelat Res., vol. 383, 2001, pp. 41-6. [PubMed])

[6] M. Al-Turk, W. K. Metcalf, "A study of the superficial palmar arteries using the Doppler Ultrasonic Flowmeter," J Anat., vol. 138, 1984, pp. 27-32. [PubMed]

[7] Z. M. Bataineh, O. Habbal, S. T. Moqattash, "Variations in the superficial palmar arch of the hand," Ital J AnatEmbryol., vol. 114(1), Jan-Mar 2009, pp. 11-20.

[8] M. Loukas, D. Holdman, S. Holdman, "Anatomical variations of the superficial and deep palmar arches," Folia Morphol (Warsz), vol. 64(2), May 2005, pp. 78-83.

[9] K. Ozkus, T. Pestelmaci, A. I. Soyluoglu, S. M. Akkin, H. I. Ozkus, "Variations of the superficial palmar arch. Folia Morphol (Warsz), vol. 57(3), 1998, pp. 251-5.

[10] E. L. Ames, M. Bissonnette, R. Acland, G. Lister, J. Firrell, "Arterial anatomy of the thumb," J Hand Surg Br., vol. 18(4), Aug. 1993, pp. 427-36.

[11] A. Sarkar, S. Dutta, K. Bal, J. Biswas, "Handedness may be related to variations in palmar arterial arches in humans," Singapore Med J., vol 53(6), Jun. 2012, pp. 409-12.

[12] O. Bilge, Y. Pinar, M. A. Ozer, F. Govsa, "A morphometric study on the superficial palamr arch of the hand," Surgical and Radiologic Anatomy, vol. 28(4), 2006, pp. 343-350

[13] P. Ruengasakuirach, N. Eizenborg, M. Fahrer, B. F. Buxton, "Surgical implications of variations in hand collateral circulation: anatomy revisited," J Thorac Cardiovas Sur, vol. 122(4), 2001, pp. 682-6. 


\section{AUTHORS' PROFILE}

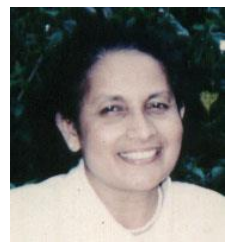

Professor L S S Salgado is the Cardre chair and Professor of Human Anatomy. Sheis currently serving as a senior lecturer in anatomy in the Faculty of medicine, University of Kelaniya. She had graduated from the University of Colombo and holds a Master in Philosophy from the University of Kelaniya. She has contributed immensely to the study of Anatomy by numerous research publications in both local and international journals. Her areas of special interest include placental studies, anatomical variations of clinical importance and medical education. She is currently residing in Kadawatha Sri Lanka. Her email is sujeesal@yahoo.com and sujeesal@kln.ac.lk

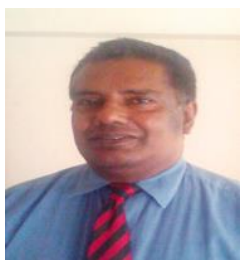

Dr R Hasan is currently the Head of the Department of Anatomy, Faculty of Medicine, University of Kelaniya. He is a senior lecturer in anatomy. He had graduated from the Faculty of Medicine, NCMC, and holds a Ph.D from the University of Ruhuna. His research interests include infertility, human evolution and morphology. He has contributed to the study of anatomy by numerous researches which have been published in both local and international journals. He is currently residing in Kandana, Sri Lanka and his email is rizvi2003@gmail.com.

\section{Perera A A M M S L:}

Dr A A M M S L Perera is a research assistant in the Department of Anatomy, Faculty of Medicine, University of kelaniya. She graduated from the University of Jaffna. She is specifically interested in clinical anatomy. She is currently residing in Negombo, Sri Lanka and her email is lavindiperera@gmail.com.

\section{Wijesundara W M R D:}

Dr W M R D Wijesundara is a research assistant in the Department of Anatomy, Faculty of Medicine, University of kelaniya. She graduated from the University of Kelaniya. She is specifically interested in neuroscience. She is currently residing in Kiribathgoda Sri Lanka and her email is rizharawijesundara@gmail.com

\section{Anuradha W K:}

Dr W K Anuradha is a research assistant in the Department of Anatomy, Faculty of Medicine, University of kelaniya. She graduated from the University of Kelaniya. 